\title{
MULTIPLIERS FROM SPACES OF TEST FUNCTIONS TO AMALGAMS
}

\section{MARIA TORRES DE SQUIRE}

(Received 28 July 1989 ; revised 13 May 1991)

Communicated by C. Sutherland

\begin{abstract}
In this paper we study the space of multipliers $M(r, s: p, q)$ from the space of test functions $\Phi_{r s}(G)$, on a locally compact abelian group $G$, to amalgams $\left(L^{p}, l^{q}\right)(G)$; the former includes (when $r=s=\infty$ ) the space of continuous functions with compact support and the latter are extensions of the $L^{p}(G)$ spaces. We prove that the space $M(\infty: p)$ is equal to the derived space $\left(L^{p}\right)_{0}$ defined by Figá-Talamanca and give a characterization of the Fourier transform for amalgams in terms of these spaces of multipliers.
\end{abstract}

1991 Mathematics subject classification (Amer. Math. Soc.): 43 A 22.

\section{Introduction}

The space of test functions $\Phi_{\infty s}(1 \leq s \leq \infty)$, on the real line, was originally defined by H. Holland [10]. The definition of $\Phi_{r s}(G)$ on a locally compact abelian group $G$, is due to Bertrandias and Dupuis [2]. The amalgam spaces $\left(L^{p}, l^{q}\right) \quad(1 \leq p, q \leq \infty)$ are Banach spaces of functions which belong locally to $L^{p}(G)$ and globally to $l^{q}$. If $p=q$ then $\left(L^{p}, l^{q}\right)$ is the usual $L^{p}(G)$ space. The purpose of this paper is to study the space $M(r, s: p, q)$ $(1 \leq r, s, p, q \leq \infty)$ of multipliers from $\Phi_{r s}(G)$ to $\left(L^{p}, l^{q}\right)(G)$. We prove the following.

(C) 1993 Australian Mathematical Society $0263-6115 / 93 \$ A 2.00+0.00$ 
1. For $1 \leq r, s, p \leq \infty$ and $1 \leq q<2$, the space $M(r, s: p, q)$ is trivial.

2. For $r=s=\infty$ and $p=q$, the space $M(\infty: p)$ is equal to the derived space $\left(L^{p}\right)_{0}$ defined by Figá-Talamanca in [6].

3. for $r=s=\infty$ and $1 \leq r, p, q \leq \infty$, the space $M(r, s: p, q)$ contains or is equal to a set of Fourier transforms of measures. In particular a measure $\mu$ is the Fourier transform of a function in $L^{p}$, for $1 \leq p \leq 2$, if and only if $\mu$ is a multiplier in $M\left(p^{\prime}: \infty\right)$.

\section{Notation and preliminary results}

Throughout this paper $G$ is a locally compact abelian group with dual group $\Gamma$. The elements of $\Gamma$ are denoted by $\hat{x}$ and we write $[x, \hat{x}]$ instead of $\hat{x}(x)(x \in G)$. As usual $C_{c}(G)\left(C_{0}(G)\right)$ is the space of continuous functions on $G$ with compact support (which vanish at infinity). For a function $f$ on $G$, we use $f^{\prime}$ to denote the reflection $f^{\prime}(y)=f(-y)$, and for $x$ in $G$, the translation operator $\tau_{x}$ is defined by $\tau_{x} f(y)=f(y-x)$. If $\mu$ is a measure on $G$, then its reflection $\mu^{\prime}$ and its translation $\tau_{x} \mu$ are defined by $\mu^{\prime}(f)=\mu\left(f^{\prime}\right)$ and $\tau_{x} \mu(f)=\mu\left(\tau_{x} f\right)\left(f \in C_{c}(G)\right)$ respectively. The pairing between a linear space $B$ and its dual $B^{*}$ is given by $\langle f, \sigma\rangle=\sigma(f)$ for $\sigma$ in $B^{*}$, and $f$ in $B$. We use $\mathbf{J}$. Stewart's definition of the amalgam spaces $\left(L^{p}, l^{q}\right)(G)=\left(L^{p}, l^{q}\right),\left(C_{0}, l^{q}\right)(G)=\left(C_{0}, l^{q}\right),\left(L^{p}, c_{0}\right)(G)=\left(L^{p}, c_{0}\right)$ $(1 \leq p, q \leq \infty)$ and the space of measures $M_{q}(G)=M_{q}(1 \leq q \leq \infty)$ [12]. We assume all the properties of inclusion, duality, and convolution product of these spaces, Hölder and Young's inequalities, and the HausdorffYoung theorem for amalgams as given in [14], and all the properties of the Segal algebra $S_{0}(G)$ given in [4] and [14]. We denote by $A$ any of the amalgams $\left(L^{p}, l^{q}\right),\left(L^{p}, c_{0}\right)(1 \leq p<\infty),\left(C_{0}, l^{s}\right)(1 \leq s \leq \infty)$. We use $H$. Feichtinger's definition of the Fourier transform as an element of $S_{0}(G)^{*}$ [4, 14 Definition 2.3]. We write $\hat{\mu}(\check{\mu})$ for the Fourier transform (inverse Fourier transform) of an element $\mu$ of $S_{0}(G)^{*}\left(S_{0}(\Gamma)^{*}\right)$. If $M$ is a subset of $S_{0}(G)^{*}$, then $M^{-}$denotes the set of Fourier transforms of element $M$. We let $\mathscr{M}_{T}$ be the space of transformable measures [1], and as usual $p^{\prime}$ is the conjugate of the number $p$. We finish this section with two preliminary results.

Proposition 1. If $\sigma \in S_{0}(G)^{*}$ and $h \in S_{0}(G)$, then $\sigma * h$ is the element of $L^{\infty}(G)$ given by $\langle f, \sigma * h\rangle=\langle f * h, \sigma\rangle$ for all $f$ in $L^{1}(G)$. Hence $\langle f, \sigma * h\rangle=\langle h, \sigma * f\rangle$ for all $f$ in $L^{1}(G)$. 
Proof. By [14, Proposition 2.8], $\sigma * h$ is in $S_{0}(G)^{*}$ and for $g$ in $S_{0}(G)$ we have that

$$
|\langle g, \sigma * h\rangle|=|\langle g * h, \sigma\rangle| \leq\|\sigma\|\|h\|_{S_{0}}\|g\|_{1} .
$$

The conclusion follows from the density of $S_{0}(G)$ in $L^{1}(G)$ [14, Proposition 2.5 and $(2.5)]$.

THeOREM 2. Let $S$ be any of the spaces $\left(L^{p}, l^{1}\right)(G) \quad(1 \leq p<\infty)$ or $\left(C_{0}, l^{1}\right)$. If $T: S \rightarrow S_{0}(G)^{*}$ is a linear bounded operator such that $T(f * g)=$ $T f * g$ for all $f$ and $g$ in $S$, then there exists a unique $\mu$ in $S_{0}(G)^{*}$ such that $T f=\mu * f$ for all $f$ in $S$.

Hence $(T f)^{-}=\sigma \hat{f}$ for all $f$ in $S$, where $\sigma=\hat{\mu}$.

Proof. The proof is essentially the same as [14, Theorem 3.2]. Observe that the functions $\lambda_{\alpha}$ defined in the proof of [14, Theorem 3.2] belongs to $S_{0}(G)\left[13\right.$, Lemma 6.4] and $S_{0}(G)$ is included in $\left(C_{0}, l^{0}\right)(G)$. The second statement follows from [14, Proposition 2.8].

\section{The space of multipliers}

The space of test functions $\Phi_{r s}(G)=\phi_{r s}(1 \leq r, s \leq \infty)$, as defined in [15, Definition 3.1] consists of continuous functions with compact support $\varphi$ such that its Fourier transform $\hat{\varphi}$ belongs to $\left(C_{0}, l^{s}\right)(\Gamma)$. It is normed by $\|\hat{\varphi}\|_{r s}($ see $[14,(1.9)])$. The duality between $\Phi_{r s}(G)$ and its Banach dual, $M_{s^{\prime}}(\Gamma)$ if $r=\infty,\left(L^{r^{\prime}}, l^{s^{\prime}}\right)(\Gamma)$ if $r$ is finite [2, $\left.\S 2 \mathrm{c}\right],[15$, Remark 3.2ii)] will be denoted by $\langle\langle\varphi, \mu\rangle\rangle$, hence

$$
\langle\langle\varphi, \mu\rangle\rangle=\int_{\Gamma} \hat{\varphi}(-\hat{x}) d \mu(\hat{x})
$$

for $\varphi \in \Phi_{r s}, \mu \in M_{s^{\prime}}(\Gamma)$ if $r=\infty, \mu \in\left(L^{r^{\prime}}, l^{s^{\prime}}\right)(\Gamma)$ if $r<\infty$. Clearly, as sets, $\Phi_{r s}$ is equal to $\Phi_{\infty}$, and as normed spaces $\Phi_{\infty}$ is continuously embedded into $\Phi_{r s}$. The space $\Phi_{\infty 1}$ is dense in $S_{0}(G)$ [123, Lemma 6.4; 5, p. 275] and it is the smallest of all the spaces $\Phi_{r s}$.

DEFINITION 3. A multiplier from $\Phi_{r s}(G)(1 \leq r, s \leq \infty)$ to the amalgam $A$ is a bounded linear operator which is translation invariant, that is, for any $x \in G, \tau_{x} T=T \tau_{x}$.

The space of multipliers will be denoted by $M(r, s: p, q)$ if $A=\left(L^{p}, l^{q}\right)$, by $M(r, s: \infty, q)$ if $A=\left(C_{0}, l^{q}\right)$, and by $M(r, s: p, \infty)$ if $A=\left(L^{p}, c_{0}\right)$. If $r=s$ or $p=q$, then we write $M(r: q)$. 
If $T$ is a multiplier from $\Phi_{r s}$ to $A$, then its adjoint $T^{\prime}$ is a bounded linear operator from $A^{*}$ to $\Phi_{r s}^{*}$, and by (1) we have for $g \in A^{*}$ and $\varphi \in \Phi_{r s}$ that

$$
\int_{\Gamma} \hat{\varphi}(-\hat{x}) d T^{\prime} g(\hat{x})=\left\langle\left\langle\varphi, T^{\prime} g\right\rangle\right\rangle=\langle T \varphi, g\rangle=\int_{G} T \varphi(x) d g^{\prime}(x) .
$$

We use this to prove that $T$ commutes with convolution.

Proposition 4. Let $T$ be in $M(r, s: p, q)(1 \leq r, s, p, q \leq \infty)$. Then for all $\varphi$ and $\psi$ in $\Phi_{r s}$ we have $T(\varphi * \psi)=T \varphi * \psi$.

Proof. Let $g$ be in $A^{*}$. By (2) and Fubini's theorem we have that

$$
\begin{aligned}
\langle T \varphi * \psi, g\rangle & =\int_{G} \int_{G} T \varphi(x-s) \psi(s) d s d g^{\prime}(x) \\
& =\int_{G} \psi(s)\left\langle\left\langle\tau_{s} \varphi, T^{\prime} g\right\rangle\right\rangle d s=\int_{\Gamma} \hat{\varphi}(-\hat{x}) \hat{\psi}(-\hat{x}) d T^{\prime} g(\hat{x}) \\
& =\left\langle\left\langle\varphi * \psi, T^{\prime} g\right\rangle\right\rangle=\langle T(\varphi * \psi), g\rangle .
\end{aligned}
$$

If $T$ is in $M(r, s: p, q)(1 \leq r, s, p, q \leq \infty), x \in G, g \in A^{*}$ and $\varphi \in \Phi_{r s}$, then as in the previous proof

$$
\left\langle\left\langle\varphi, T^{\prime} \tau_{x} g\right\rangle\right\rangle=\left\langle T \tau_{x} \varphi, g\right\rangle=\left\langle\left\langle\varphi,[x, .] T^{\prime} g\right\rangle\right\rangle .
$$

Hence

$$
T^{\prime} \tau_{x} g=[x, .] T^{\prime} g
$$

If $F$ is the Fourier transform on $\Phi_{r s}^{*}$ and $T$ is multiplier in $M(r, s$, : $p, q) \quad(1 \leq r, s, p, q \leq \infty)$, then by (2), Proposition 4, and [14, Proposition $2.5,2.8$ ] the composition of $F$ and $T^{\prime}$ is a bounded linear operator which commutes with convolution. That is, for $g$ and $f$ in $\left(L^{p^{\prime}}, l^{1}\right)(G)$ if $1<p \leq \infty$ and in $\left(C_{0}, l^{1}\right)(G)$ if $p=1$ we have that

$$
F T^{\prime}(f * g)=F T^{\prime} f * g .
$$

This together with Theorem 2, Proposition 4, and [14, Remark 2.4 ii)] implies that there exists $\mu \in S_{0}(G)^{*}$, hence a unique $\sigma \in S_{0}(\Gamma)^{*}$, such that

$$
\begin{gathered}
F T^{\prime} f=\mu * f \\
f^{\prime} f=\sigma \hat{f}=(\mu * f)^{-} .
\end{gathered}
$$

Moreover, since $S_{0}(G)$ is included in $\left(L^{p^{\prime}}, l^{1}\right)$ and $\left(C_{0}, l^{1}\right)$ we have by Proposition $1,(5)$, and $[14,(1.9)]$ that $\mu * f$ is a transformable measure for 
all $f$ in $S_{0}(G)$. Hence by [1, Corollary 3.1], if $\varphi \in \Phi_{r s}(G)$, then $\hat{\varphi}$ belongs to $L^{1}\left(T^{\prime} f\right)$ and therefore

$$
\int_{G} \varphi(x) \mu * f(x) d x=\int_{\Gamma} \hat{\varphi}(-\hat{x}) d\left(T^{\prime} f\right)(\hat{x}) .
$$

By (1) and Proposition 1 we conclude that

$$
\langle\mu * \varphi, f\rangle=\langle T \varphi, f\rangle
$$

for all $f$ in $S_{0}(G)$.

By the density of $S_{0}(G)$ in $A$, and [14, Theorem 1.4], we conclude that for all $\varphi \in \Phi_{r s}$

$$
T \varphi=\mu * \varphi .
$$

From (4) and (5) and the fact that $\Phi_{r s}$ is included in the amalgams $\left(L^{p^{\prime}}, l^{1}\right)$ and $\left(C_{0}, l^{1}\right)$ we have that

$$
T \varphi=F T^{\prime} \varphi \text { and }(T \varphi)^{\curlywedge}=T^{\prime} \varphi \text { for all } \varphi \in \Phi_{r s} .
$$

Proposition 5. Let $T$ be in $M(r, s: p, q)(1 \leq r, s, p, q \leq \infty)$. The functional $\sigma$ in $S_{0}(\Gamma)^{*}$ associated to $T^{\prime}$ in (5) belongs to $M_{\infty}(\Gamma)$. Moreover, $\sigma$ belongs to

1. $\left(L^{1}, l^{\infty}\right)(\Gamma)$ if either $r$ is finite or $1 \leq q \leq 2$.

2. $M_{2}(\Gamma)$ if $r=s=\infty$.

3. $\left(L^{1}, l^{2}\right)(\Gamma)$ if $r=s=\infty$ and $1 \leq q \leq 2$.

4. $\left(L^{r^{\prime}}, l^{\infty}\right)(\Gamma)$ if $r=2$ and $r$ is finite.

5. $\left(L^{q^{\prime}}, l^{\infty}\right)(\Gamma)$ if $1 \leq q \leq 2$ and $2 \leq p, s \leq \infty$.

Proof. We take $E$ a compact subset of $\Gamma, h$ a continuous function with compact support contained in $E$, and $g$ a function in $\Phi_{\infty 1}(G)$ such that $\hat{g}$ is in $C_{c}(G)$ and $\hat{g} \equiv 1$ on $E[12$, Theorem 3.1]. By [14, (2.6] we have that

$$
\begin{aligned}
|\langle h, \sigma\rangle| & =|\langle h \hat{g}, \sigma\rangle|=|\langle h, \sigma \hat{g}\rangle|=\left|\left\langle h, T^{\prime} g\right\rangle\right| \\
& \leq\left\|T^{\prime} g\right\|_{r^{\prime} s}\|h\|_{r s} \leq C_{E}\|h\|_{\infty}
\end{aligned}
$$

where $C_{E}$ is a constant depending on $E$.

Therefore $\sigma$ is a measure of $\Gamma$ by [5, Theorem $B 1 ; 11$, Theorem 5.1.4].

Now for $\beta$ in $I$ the function $\tau_{\beta} g$ is equal to one on $L_{\beta}$, ( $I$ and $L_{\beta}$ as in [14, Remark 1.3]) and $T^{\prime}([\beta,] g)=.\sigma \tau_{\beta} \hat{g}$ belongs to $M_{s^{\prime}}(\Gamma)[14,(1.9)]$, 
hence

$$
\begin{aligned}
|\sigma|\left(L_{\beta}\right)^{s^{\prime}} & =\left[\int_{L_{\beta}}\left|\tau_{\beta} \hat{g}\right|(\hat{x}) d|\sigma|(\hat{x})\right]^{s^{\prime}} \\
& =\left\|\sigma \tau_{\beta} \hat{g}\right\|_{s^{\prime}}^{s^{\prime}} \leq\left\|T^{\prime}\right\|^{s^{\prime}}\|[\beta, .] g\|_{p^{\prime} q^{\prime}}^{s^{\prime}}=\left\|T^{\prime}\right\|^{s^{\prime}}\|g\|_{p^{\prime} q^{\prime}}^{s^{\prime}}
\end{aligned}
$$

and therefore $\sigma$ is a measure in $M_{\infty}(\Gamma)$. To prove 1 we take a compact subset $K$ of $\Gamma$ with Haar measure zero, and a function $\varphi$ in $\Phi_{r s}(G)$ such that $\hat{\varphi} \equiv 0$ on $K$. If $r$ is finite, then $\sigma \hat{\varphi}=T^{\prime} \varphi$ is a function in $\left(L^{r^{\prime}}, l^{s^{\prime}}\right)(\Gamma)$ and we have that

$$
\sigma(K)=\int_{K} \hat{\varphi}(\hat{x}) d \sigma(\hat{x})=\int_{K} T^{\prime} \varphi(\hat{x}) d(\hat{x})=0 .
$$

Hence $\sigma$ is absolutely continuous with respect to the Haar measure on $\Gamma$ and we conclude from [3, Chapter V] that $\sigma$ belongs to $\left(L^{1}, l^{\infty}\right)(\Gamma)$. If $1 \leq q \leq 2$, then by (6) and [14, Proposition 2.8, Remark 2.7] we have that $\sigma \hat{\varphi}=(T \varphi)^{\wedge}$ is a function on $\Gamma$. As before this implies that $\sigma$ is in $\left(L^{1}, l^{\infty}\right)(\Gamma)$.

To prove 3 we note that $\Phi_{\infty}$ is equal to $\Phi_{\infty 2}$ as sets, and by (5), for any $\varphi \in \Phi_{\infty}$, the measure $\sigma \hat{\varphi}$ belongs to $M_{1}(\Gamma)$ that is, $\sigma$ is a Fourier multiplier on $\Phi_{\infty 2}$ and by [15, Theorem 6.15], $\sigma$ is in $M_{2}(\Gamma)$. Part 4 follows from 1 and 2 .

Now, if $r$ is finite and $s=2$, then $\sigma \hat{\varphi}$ belongs to $\left(L^{r^{\prime}}, l^{2}\right)(\Gamma)$ for all $\varphi \in \Phi_{r 1}$. Hence $\nu \sigma \hat{\varphi}$ is in $L^{1}(\Gamma)$ for any $\nu$ in $\left(l^{r}, l^{2}\right)(\Gamma)$.

Again by [15, Theorem 6.1], $\nu \sigma$ belongs to $\left(L^{1}, l^{2}\right)(\Gamma)$ and by the converse of Hölder's inequality $\sigma$ is in $\left(L^{r^{\prime}}, l^{\infty}\right)(\Gamma)$.

Part 5 is similar to 4 ; note that $\sigma \hat{\varphi}=(T \varphi)^{-}$belongs to $\left(L^{q^{\prime}}, l^{2}\right)(\Gamma)$ for all $\varphi \in \Phi_{r s}$.

From (6) and Proposition 2.4 we see that $M(r, s: p, q)(1 \leq r, s, p, q \leq$ $\infty)$ is isometrically isomorphic to the set of $\mu \in S_{0}(G)^{*}$ such that $\hat{\mu}$ is in $M_{\infty}(\Gamma)$ if $r=\infty$ and in $\left(L^{1}, l^{\infty}\right)(\Gamma)$ if $r$ is finite, and norm equal to

$$
\|\| \mu \mid \|=\sup \left\{\|\mu * \varphi\|_{p q} \mid \varphi \in \Phi_{r s},\|\hat{\varphi}\|_{r s} \leq 1\right\} .
$$

We now use the concept of set of uniqueness, to show that for $1 \leq q<2$, $(1 \leq r, s, p \leq \infty)$ the space $M(r, s: p, q)$ is trivial (cf. [6, Theorem 3]).

DEFinition 6. A subset $E$ of $\Gamma$ is a set of uniqueness for $\left(L^{p}, l^{q}\right)(G)$ $(1 \leq p, q \leq \infty)$, if for any $f$ in $\left(L^{p}, l^{q}\right)(G)$ such that $\hat{f}$ vanishes outside $E$, then $f \equiv 0$.

Sets of uniqueness for $\left(L^{p}, l^{q}\right)(G) \quad(1 \leq p, q \leq 2)$ always exists [8, page 133], and also for $\left(L^{r}, l^{q}\right)(G) \quad(2 \leq r \leq \infty, 1 \leq q \leq 2)$ because $\left(L^{r}, l^{q}\right) \subset$ $\left(L^{p}, l^{q}\right)$ for $1 \leq p \leq 2 \leq r \leq \infty$. 
TheOREM 7. If $1 \leq p \leq \infty, 1 \leq q<2$ and $f$ is a function on $\Gamma$ such that $f \varphi$ belongs to $\left(L^{p}, l^{q}\right)(G)^{\wedge}$ for all $\varphi \in C_{c}(\Gamma)$. Then $f \equiv 0$ locally almost everywhere.

Proof. Suppose that $f$ does not vanish locally almost everywhere. Then there exists a compact set $K$ of nonnegative measure such that $f$ does not vanish almost everywhere on $K$. Let $\psi$ be a continuous function with compact support such that $\psi \equiv 1$ on $K$. Then $\psi f$ does not vanish locally almost everywhere. Since $\psi \varphi$ is in $C_{c}(\Gamma)$ for all $\varphi \in C_{c}(\Gamma)$, it follows that $\psi \varphi f$ belongs to $\left(L^{p}, l^{q}\right)(G)^{\wedge}$ for all $\varphi \in C_{c}(\Gamma)$. Thus without loss of generality we can assume that $f$ vanishes off some compact set $K$ of nonnegative measure.

If $p=q=1$, then $\varphi f$ is in $L^{1}(G)^{\wedge}$ for all $\varphi \in C_{c}$, so $\varphi f=\hat{g}$ for some $g \in L^{1}(G)$. Since $\varphi f$ is in $L_{c}^{\infty}$ and $L_{c}^{\infty} \subset\left(L^{2}, l^{1}\right)$, the function $g$ belongs to $L^{1} \cap\left(L^{\infty}, l^{2}\right)$, then by the Riesz-Thorin theorem [13, Theorem 5.6; 10 , Theorem 5], we have that $g$ is in $\left(L^{p}, l^{q}\right)$ for some fixed $1<p<\infty$, $1<q<2$, so we can further assume that $\varphi f$ belongs to $\left(L^{p}, l^{q}\right)(G)^{\wedge}$ for some fixed $1<p<\infty, 1<q<2$.

If $p=\infty$ and $q=1$, then as above $g \in\left(L^{\infty}, l^{1}\right)$ and $\varphi f$ is in $L^{2}(\Gamma)$, so $g$ is in $\left(L^{\infty}, l^{1}\right) \cap L^{2}$. By the same argument we can assume that $\varphi f$ is in $\left(L^{p}, l^{q}\right)(G)^{\wedge}$ for some fixed $2<p<\infty, 1<q<2$.

If $\varphi$ is a function in $C_{c}(\Gamma)$ such that $\varphi \equiv 1$ on $K$, then $\varphi f=f$, hence $f$ is in $\left(L^{p}, l^{q}\right)(G)^{-}$and therefore $f$ is a function in $L^{q^{\prime}}$ with compact support, because $f$ vanishes off $K$. Thus $f$ belongs to $L^{2}(\Gamma)$.

Let $S$ be the map defined on $C_{c}(\Gamma)$ by $(S \varphi)^{-}=\varphi f$. An application of the Closed Graph Theorem shows that $S$ restricted to $C_{c}(E)$, for $E$ a compact subset of $\Gamma$, is continuous. Now we take $E$ a compact subset of $\Gamma$ and $\left\{\varphi_{n}\right\}$ a sequence in $C_{c}(\Gamma)$ such that $\varphi_{n} \equiv 1$ on $E$ for all $n$, $0 \leq \varphi_{n}(\hat{x}) \leq 1$ for all $\hat{x}$ in $\Gamma$, and the support of each $\varphi_{n}$ is equal to $E$, with $E_{n+1} \subset E_{n}$ and $E=\cap E_{n}$. Hence $\left\{\varphi_{n}\right\} \subset C_{c}\left(E_{1}\right)$ and converges pointwise to $\chi_{E}$, the characteristic function of $E$. Since $E_{n+1} \subset E_{n}$ for all $n$, there is a constant $C_{E}$, depending on $E_{1}$ such that $\left\|\varphi_{n}\right\| \leq C_{E}$ for all $n$. Hence $\left\|S \varphi_{n}\right\|_{p q} \leq\left\|\varphi_{n}\right\|_{\infty} \leq C_{E}$; that is, $\left\{S \varphi_{n}\right\}$ is a normed subset of $\left(L^{p}, l^{q}\right)$, and therefore it has a weakly convergent subset $\left\{S \varphi_{k}\right\}$. Let $g$ in $\left(L^{p}, l^{q}\right)$ be such that $\lim \left\langle S \varphi_{k}, h\right\rangle=\langle g, h\rangle \quad\left(h \in\left(L^{p^{\prime}}, l^{q^{\prime}}\right)(\Gamma)\right)$. Since $\left|\varphi_{k} f\right| \leq|f|$ on $\Gamma$, we have that for $h \in C_{c}(\Gamma)$

$$
\left\langle S \varphi_{k}, h\right\rangle=\lim \left\langle\left(S \varphi_{k}\right)^{\uparrow}, k\right\rangle=\lim \left\langle\varphi_{k} f, \hat{h}\right\rangle=\left\langle\chi_{E}, \hat{h}\right\rangle=\left\langle\left(\chi_{E} f\right)^{-}, h\right\rangle .
$$

We conclude that $\left(\chi_{E} f\right)^{\wedge}=g$. But if $E$ is a subset for $K$ and a set of 
uniqueness for $\left(L^{p}, l^{q}\right)$, then this is a contradiction because $\chi_{E} f$ does not vanish almost everywhere on $E$.

Proposition 8. If $\mu$ is a multiplier in $M(r, s: p, q)$ for $1 \leq r, s, p, \leq$ $\infty, 1 \leq q<2$, then

$$
\hat{\mu} h \in\left(L^{p}, l^{q}\right)(G)^{\wedge} \text { for all } h \in C_{c}(\Gamma) .
$$

Proof. By Proposition 5, $\hat{\mu}$ is a function in $\left(L^{1}, l^{\infty}\right)(\Gamma)$. By $\left.[2, \S 2, \mathrm{c})\right]$ for $h \in C_{c}(\Gamma)$, there is a sequence $\left\{h_{n}\right\}$ in $\Phi_{\infty 1}(G)$ such that $\lim \left\|\hat{h}_{n}-h\right\|_{\infty 1}=0$. Since $\left\|\mu * h_{n}\right\|_{p q} \leq\|\| \mu\|\| \hat{h}_{n} \|_{\infty 1}$, the sequence $\left\{\mu * h_{n}\right\}$ is Cauchy in $\left(L^{p}, l^{q}\right)(\Gamma)$, so $\lim \left\|\mu * h_{n}-g\right\|_{p q}=0$ for some $g$ in $\left(L^{p}, l^{q}\right)(G)$. Since $S_{0}(G)$ is a subspace of $\left(C_{0}, l^{1}\right)(G)$, the pointwise product of $\psi$ and $h_{n}-h$ belongs to $L^{1}(\hat{\mu})=\left(C_{0}, l^{1}\right)(G),[13$, Proposition 4.1]. Hence for $\psi$ in $S_{0}(G)$

$$
\begin{aligned}
\left|\left\langle\psi, \hat{\mu}\left(\hat{h}_{n}\right)\right\rangle\right| & =\left|\left\langle\psi\left(\hat{h}_{n}-h\right), \hat{\mu}\right\rangle\right| \\
& \leq\|\hat{\mu}\|_{\infty}\left\|\psi\left(\hat{h}_{n}-h\right)\right\|_{\infty 1} \leq\|\hat{\mu}\|_{\infty}\|\psi\|_{\infty}\left\|\hat{h}_{n}-h\right\|_{\infty 1} \\
& \leq\|\hat{\mu}\|_{\infty}\|\psi\|_{\infty k^{\prime}}\left\|\hat{h}_{n}-h\right\|_{\infty 1}
\end{aligned}
$$

where $k$ is equal to $p^{\prime}$ if $1 \leq p \leq 2$ and to 2 if $2 \leq p \leq \infty$. By the density of $S_{0}(\Gamma)$ in $\left(C_{0}, l^{k^{\prime}}\right)(\Gamma)$ we conclude that $\hat{\mu}\left(\hat{h}_{n}-h\right)$ is a function in $M_{k}(\Gamma)$ and therefore $\lim \left\|\hat{\mu}\left(\hat{h}_{n}-h\right)\right\|_{1 k}=0$ [14, page 125]. Since $\hat{h}-\hat{g}$ belongs to $\left(L^{q^{\prime}}, l^{k}\right)(\Gamma)$ and $\hat{\mu} \hat{h}_{n}=\left(\mu * h_{n}\right)^{-}$(cf. (5) and (6)) we have by the continuity of the Fourier transform that

$$
\begin{aligned}
\|\hat{\mu} h-\hat{g}\|_{1 k} & \leq\left\|\hat{\mu} \hat{h}_{n}-\hat{\mu} h\right\|_{1 k}+\left\|\hat{\mu} \hat{h}_{n}-\hat{g}\right\|_{1 k} \\
& \leq\left\|\hat{\mu}\left(\hat{h}_{n}-h\right)\right\|_{1 k}+\left\|\hat{\mu} \hat{h}_{n}-\hat{g}\right\|_{q^{\prime} k} \\
& \leq\left\|\hat{\mu}\left(\hat{h}_{n}-h\right)\right\|_{1 k}+C\left\|\mu * h_{n}-g\right\|_{p q}
\end{aligned}
$$

where $C$ is a constant depending on $G, p$ and $q$. This implies that $\hat{\mu} h=\hat{g}$.

Corollary 9. The space $M(r, s: p, q)$ for $1 \leq r, s, p \leq \infty, 1 \leq q<2$ is trivial.

Proof. Theorem 7, Proposition 8, and the inclusions $M(r, s: p, q) \subset$ $M(\infty, r: p, q) \subset M(\infty, 1: p, q)$.

This last result is for any locally compact abelian group, and this improves [11, Theorems 4.6.5 and 4.6.6] because as we will see in the next section, the derived space $\left(L^{p}\right)_{0}$ defined in [6] is equal to $M(\infty: p)$. 


\section{Special infinite cases}

In this section we give necessary and sufficient conditions for an element of $S_{0}(G)^{*}$ to be a multiplier.

Proposition 10. Let $\mu$ be an element of $S_{0}(G)^{*}$ with the Fourier transform $\hat{\mu}$ in $\left(L^{r^{\prime}}, l^{\infty}\right)(\Gamma)$ for $1 \leq r<\infty\left(\right.$ in $\left.M_{\infty}(\Gamma)\right)$. If $\hat{\mu} h$ is an element of $A$ for each $h$ in $\left(L^{r}, l^{s}\right)(\Gamma)$ in $\left.\left(C_{0}, l^{s}\right)(\Gamma)\right)(1 \leq s \leq \infty)$, then $\mu$ belongs to $M(r, s: p, q)($ to $M(\infty, s: p, q))(1 \leq p, q \leq \infty)$.

Proof. We define the map $S$ on $\left(L^{r}, l^{s}\right)(\Gamma)$ by $(S h)^{-}=\hat{\mu} h$. Let $\left\{h_{n}\right\}$ be a sequence in $\left(L^{r}, l^{s}\right)$ such that $\lim \left\|h_{n}-h\right\|_{r s}=0$ and suppose that $\lim \left\|h_{n}-g\right\|_{A}=0$. For $\psi \in S_{0}(G)$ we have that

$$
\begin{aligned}
\left|\left\langle\psi,(S h)^{-}-\hat{g}\right\rangle\right| & \leq\left|\left\langle\psi,\left(S h_{n}\right)^{\hat{-}}-(S h)^{\hat{\gamma}}\right\rangle\right|+\left|\left\langle\psi,\left(S h_{n}\right)^{-}-\hat{g}\right\rangle\right| \\
& \leq\left|\left\langle\psi, \hat{\mu} h_{n}-\hat{\mu} h\right\rangle\right|+\left|\left\langle\hat{\psi}, S h_{n}-g\right\rangle\right| \\
& \leq\left|\left\langle\psi\left(h_{n}-h\right), \hat{\mu}\right\rangle\right|+\|\hat{\psi}\|_{A^{*}}\left\|S h_{n}-g\right\|_{A} \\
& \leq\|\hat{\mu}\|_{r^{\prime} \infty}\|\psi\|_{\infty s^{\prime}}\left\|h_{n}-h\right\|_{r s}+\|\hat{\psi}\|_{A^{*}}\left\|S h_{n}-g\right\|_{A^{\prime}} .
\end{aligned}
$$

From [14, Remark 2.4 iii)], the density of $S_{0}(G)$ in $A$, and the Closed Graph Theorem, the map $S$ is continuous. Now, if $\psi \in \Psi_{r s}$, then by [14, Proposition 2.8] we have that

$$
\|\mu * \psi\|_{A}=\|S \hat{\psi}\|_{A} \leq\|S\|\|\hat{\psi}\|_{r s} .
$$

The proof for $r=\infty$ is similar.

REMARKs. The space $\mathscr{R}\left(\Phi_{r s}\right) \quad(1 \leq r, s \leq \infty)$ of resonant classes of measures relative to $\Phi_{r s}[15$, Definition 3.3] consists of transformable measures whose Fourier transform belongs to $\left(L^{r^{\prime}}, l^{s^{\prime}}\right)(\Gamma)$ if $1 \leq r<\infty$ to $M_{\infty s^{\prime}}(\Gamma)$ if $r=\infty$.

From Proposition 10, Corollary 7, [15, Corollary 3.5; 1, Theorem 2.5] we have that

1. if $\mu \in \mathscr{M}_{T}$ with $\hat{\mu} h \in \mathscr{R}\left(\Phi_{p^{\prime} q^{\prime}}\right) \quad(1<p \leq \infty, 1 \leq q<2)$ for each $h \in\left(C_{0}, l^{1}\right)(\Gamma)$, then $\mu \equiv 0$.

2. if $f \in\left(L^{p}, l^{q}\right)(G) \quad(1 \leq q<2,1 \leq p \leq \infty)$ and $\hat{f} h \in\left(L^{p}, l^{q}\right)(\Gamma)^{-}$ for each $h \in\left(C_{0}, l^{1}\right)(\Gamma)$, then $f \equiv 0$. That is, the subspace of $\left(L^{p}, l^{q}\right)(G)$ invariant under the product of Fourier transforms by elements of $\left(C_{0}, l^{2}\right)(\Gamma)$ is trivial. 
When $p=q$, this improves Figá-Talamanca's result in [6] because $\left(C_{0}, l^{1}\right)$ is a subspace of $C_{0} \cap L^{1}$.

TheOREM 11. An element $\mu \in S_{0}(G)^{*}$ is a multiplier in $M(r, s: p, \infty)$ $(1 \leq r, s, p \leq \infty)$ if and only if for each $g$ in $\left(L^{p^{\prime}}, l^{1}\right)(G)$ there exists a measure $\nu_{g}$ in $M_{s^{\prime}}(\Gamma)$ if $r=\infty$ and in $\left(L^{r^{\prime}}, l^{s^{\prime}}\right)(\Gamma)$ if $r$ is finite, such that $\mu * g=\hat{\nu}_{g}$.

Proof. The necessity part follows from (4) and (6). We now assume that $r$ is finite and let $R$ be the Segal algebra $\left(L^{p}, l^{1}\right)$ if $1<p \leq \infty$ and $\left(C_{0}, l^{1}\right)$ if $p=1$. We define the map $S$ on $R$ by $S g=\nu_{g}$. As in the previous proposition an application of the Closed Graph Theorem shows that $S$ is continuous. By Proposition 1 and the fact that $(\mu * g)^{\wedge}=S g\left(g \in S_{0}(G)\right)$ [14, Remark 2.4ii)] the convolution $\mu * g$ is a transformable measure. Hence by [1, Corollary 3.1] for $\psi \in \Phi_{r s}(G)$ and $g \in S_{0}(G)$ we have that

$$
\begin{aligned}
|\langle g, \mu * \psi\rangle| & =|\langle\psi, \mu * g\rangle|=|\langle\psi,(\mu * g) \hat{}\rangle| \\
& =|\langle\hat{\psi}, S g\rangle| \leq\|S g\|_{r^{\prime} s^{\prime}}\|\hat{\psi}\|_{r s} \leq\|S\|\|g\|_{R}\|\hat{\psi}\|_{r s} .
\end{aligned}
$$

Since $S_{0}(G)$ is dense in $R$ and $\hat{\mu} \hat{\psi}=S \psi$ for all $\psi \in S_{0}(G)$, we conclude as in the proof of Proposition 5 that $\mu$ is a multiplier. The case $r=\infty$ is similar.

By [14, Theorem 6.2] we see that if $T \in M(\infty: p, q)$, then the element $\mu$ associated to $F T^{\prime}$ in (4) belongs to $\left(L^{p}, l^{q}\right)(\Gamma)$. Hence by (6), $M(\infty: p, q) \subset\left(L^{p}, l^{q}\right)$, but this is not always the case, as we will see in $\S 4$. The next theorem gives necessary and sufficient conditions for a function in $\left(L^{p}, l^{q}\right)(G)$ to be a multiplier.

THEOREM 12. A function $f$ in $\left(L^{p}, l^{q}\right)(G)$ belongs to $M(r, s: p, q)$ $(1 \leq r, s, p, q \leq \infty)$ if and only if for each $g$ in $\left(L^{p^{\prime}}, l^{q^{\prime}}\right)(G)$, there exists a unique measure $\nu_{g}$ in $M_{s^{\prime}}(\Gamma)$ if $r=\infty$ in $\left(L^{r^{\prime}}, l^{s^{\prime}}\right)(\Gamma)$ if $r$ is finite, such that $f * g=\check{\nu}_{g}$.

Proof. Suppose that $f$ is in $M(r, s: p, q)$ and define the function $F$ on $\Phi_{r s}(G)$ by $F(\psi)=f * g * \psi(0)$. Clearly $F$ is linear and $|F(\psi)| \leq$ \|\|$f\|\|\|\|_{p^{\prime} q}\|\hat{\psi}\|_{r s}$. By [15, Remark 3.2] there exists $\nu_{g}$ in $\Phi_{r s}(G)^{*}$ such that $\langle\psi, f * g\rangle=\left\langle\left\langle\psi, \nu_{g}\right\rangle\right\rangle$. This implies that $f * g$ is transformable and $(f * g)^{\wedge}=\nu_{g}[1, \S 2]$, hence $f * g=\hat{\nu}_{g}[14$, Remark 2.4 ii)]. To prove the converse we define the function $S$ on $\left(L^{p^{\prime}}, l^{q^{\prime}}\right)(G)$ by $(S g)^{-}=\nu_{g}$ and, as 
in Theorem 11, the function $S$ is continuous. Now for $\psi \in \Phi_{r s}$ we have that

$$
\|f * \psi\|_{p q}=\sup \left\{\mid\langle g, f * \psi\rangle \| g \in B \text { and }\|g\|_{B} \leq 1\right\}
$$

where $B$ is the amalgam $\left(L^{p^{\prime}}, l^{q^{\prime}}\right)(G)$ if $1<p, q \leq \infty\left(C_{0}, l^{q^{\prime}}\right)$ if $p=1$, $1<q \leq \infty$ or $\left(L^{p}, c_{0}\right)$ if $1<p \leq \infty, q=1$. Since

$$
|\langle g, f * \psi\rangle|=|\langle\psi, f * g\rangle|=\mid\langle\psi,(S g)\rangle \|\left\langle\hat{\psi}, S g\langle| \leq\|S\|\|g\|_{B}\|\hat{\psi}\|_{r^{\prime} s^{\prime}}\right.
$$

we conclude as in the proof of Theorem 11 that $f$ is a multiplier.

Remark. From Theorem 12 and [6, Lemma 1] the space $\left(L^{p}\right)_{0}$ is equal to $M(\infty: p)$. Moreover $M(\infty: p, q) \subset\left(L^{p}, l^{q}\right) \cap \mathscr{M}_{\mathscr{T}}$ for $1 \leq p, q \leq \infty$ $[1$, Theorem 2.3].

\section{Spaces of Fourier transform of measures}

In [6, §4] Figá-Talamanca showed that $\left(L^{p^{\prime}}\right)^{\vee} \subset M(\infty: p) \quad(2 \leq p \leq \infty)$ and $M_{1}^{\vee}=M(\infty: \infty)$. Similarly, in this section, we consider the problem of finding a space of measures $M$ such that $M^{\vee} \subset M(r, s: p, q)$.

THEOREM 13. 1 . Let $2 \leq p, q \leq \infty, 1 \leq s \leq \infty, 1 \leq r<\infty$. If $1 / x=$ $1 / q+1 / r \leq 1$ and $1 / y=1 / p+1 / s \leq 1$, then $\left(L^{x^{\prime}}, l^{y^{\prime}}\right)(\Gamma)^{\vee} \subset M(r, s: p, q)$ and $\left(L^{r^{\prime}}, l^{s^{\prime}}\right)(\Gamma)^{\vee}=M(r, s: \infty)$.

2. Let $2 \leq q<\infty, 2 \leq p \leq \infty, 1 \leq s \leq \infty$. If $y$ is as in part 1 , then $\left(L^{q^{\prime}}, l^{y^{\prime}}\right)(\Gamma)^{\vee} \subset M(\infty, s: p, q), M_{y^{\prime}}(\Gamma)^{\vee} \subset M(\infty, s: p, \infty)$ and $M_{s^{\prime}}(\Gamma)^{\vee}=M(\infty, s: \infty)$.

3. Let $2 \leq q \leq \infty, 1 \leq p \leq 2,1 \leq r<\infty, 1 \leq s \leq \infty$. If $x$ is as in part 1 and $1 / y=1 / 2+1 / s \leq 1$, then $\left(L^{x^{\prime}}, l^{y^{\prime}}\right)(\Gamma)^{\vee} \subset M(r, s: p, q)$.

4. Let $2 \leq q<\infty, 1 \leq p \leq 2,1 \leq s \leq \infty$. If $y$ is as in part 3, then $\left(L^{q^{\prime}}, l^{y^{\prime}}\right)(\Gamma)^{\vee} \subset M(\infty, s: p, q), M_{1}(\Gamma)^{\vee} \subset M(\infty, s: p, \infty)$ and $\left(L^{1}, l^{2}\right)(\Gamma)^{\vee} \subset M(\infty: p, \infty)$.

Proof. 1. Let $f \in\left(L^{x^{\prime}}, l^{y^{\prime}}\right)(\Gamma), h \in S_{0}(G)$ and $\psi \in \Phi_{r s}(G)$. By [14, Definition 2.3 and (2.5)] we have that

$$
\begin{aligned}
|\langle h, \check{f} * \psi\rangle| & =|\langle\check{h} \check{\psi}, f\rangle| \leq\|f\|_{x^{\prime} y^{\prime}}\|\check{h} \check{\psi}\|_{x y} \\
& \leq\|f\|_{x^{\prime} y^{\prime}}\|\hat{h}\|_{p q}\|\hat{\psi}\|_{r s} \leq\|f\|_{x^{\prime} y^{\prime}} C\|h\|_{p^{\prime} q^{\prime}}\|\check{\psi}\|_{r s}
\end{aligned}
$$

where $C$ is a constant depending on $G, p$ and $q$, given by the HausdorffYoung theorem for amalgams [14, Remark 2.7]. 
Since $S_{0}(G)$ is dense in $\left(L^{p^{\prime}}, l^{q^{\prime}}\right)$ we conclude by [14, Remark 2.4 ii)] that $f$ is in $M(r, s: p, q)$ and

$$
\|\| f \mid\|\leq C\| f \|_{x^{\prime} y^{\prime}}
$$

The inclusion $\left(L^{r^{\prime}}, l^{s^{\prime}}\right)(\Gamma)^{\vee} \subset M(r, s: \infty)$ is proven in a similar manner.

If $f$ is in $M(r, s: \infty)$, then clearly the map $F(\psi)=\langle\psi, f\rangle$ is a functional on $\Phi_{r s}(G)$. Hence by [15, Remark 3.2 ii)] there exists $\mu \in\left(L^{r^{\prime}}, l^{s^{\prime}}\right)(\Gamma)$ such that $\langle\psi, f\rangle=\langle\langle\psi, \mu\rangle\rangle=\langle\psi, \breve{\mu}\rangle$ for all $\psi \in \Phi_{r s}(G)$, and in particular for all $\psi \in \Phi_{\infty 1}$. Since $\Phi_{\infty 1}$ is dense in $S_{0}(G)$, we conclude that $f=\check{\mu}$.

The proofs for 2, 3, and 4 are similar.

The amalgam $\left(L^{1}, l^{2}\right)$ is the biggest space of functions whose Fourier transform is also a function [9]. Thus we see from Theorem 13, that if $y^{\prime}>2$, then $M(r, s: p, q)$ contains elements of $S_{0}(G)^{*}$ which are not functions. We will show that for certain values of $p, q, r, s$, the space $M(r, s: p, q)$ is included in an amalgam space, and contains a space of Fourier transforms. The constant which appears in the next result is given by the Hausdorff-Young theorem.

Corollary 14. 1. If $2 \leq q<\infty$ and $2 \leq p \leq \infty$, then

(a) $\left(L^{q^{\prime}}, l^{p ;}\right)(\Gamma)^{\vee} \subset M(\infty: p, q) \subset\left(L^{p}, l^{q}\right)(G)$ and $\|f\|_{p q} \leq\|\check{f}\| \leq \leq C\|f\|_{q^{\prime} p^{\prime}}$, (b) $M_{p^{\prime}}(\Gamma)^{\vee} \subset M(\infty: p, \infty) \subset\left(L^{p}, l^{\infty}\right)(G)$ and $\|\check{\mu}\|_{p \infty} \leq\|\ddot{\mu}\| \mid C\|\mu\|_{p^{\prime}}$,

where $C$ is a constant depending on $G, p$ and $q$.

2. If $2 \leq q<\infty$ and $1 \leq p \leq 2$, then

(a) $\left(L^{q^{\prime}}, l^{2}\right)(G)^{\vee} \subset M(\infty: p, q) \subset\left(L^{2}, l^{q}\right)(G)$ and $\|\check{f}\|_{2 q} \leq\|\check{f}\| \mid \leq$ $C\|f\|_{q^{\prime} 2}$,

(b) $M_{2}(\Gamma)^{\vee} \subset M(\infty: p, \infty) \subset\left(L^{2}, l^{\infty}\right)(G)$ and $\|\check{\mu}\|_{2 \infty} \leq\|\check{\mu}\| \leq \leq$ $C\|\mu\|_{2}$,

where $C$ is a constant depending on $G$ and $q$.

3. If $2 \leq r, s \leq \infty, 2 \leq q<\infty$ and $1 / x=1 / q+1 / s \leq 1$, then $\left(L^{x^{\prime}}, l^{s^{\prime}}\right)(\Gamma)^{\vee} \subset M(r, s: \infty, q) \subset\left(L^{s}, l^{r}\right)(G)$ and $\|\check{f}\|_{s r} \leq C\|\| \check{f} \| \leq$ $C^{2}\|f\|_{x^{\prime} s^{\prime}}$ where $C$ is a constant depending on $G, r$, and $s$.

4. If $1 \leq r \leq 2 \leq s \leq \infty, 2 \leq q<\infty$ and $x$ is as part 3), then $\left(L^{x^{\prime}}, l^{s^{\prime}}\right)(\Gamma)^{\vee} \subset M(r, s: \infty, q) \subset\left(L^{p}, l^{q}\right)(G) s$ and $\|\check{f}\|_{s 2} \leq C\|\check{f}\| \leq$ $C^{2}\|f\|_{s x}$ where $C$ is a constant depending on $G$ and $s$.

5. If $2 \leq s \leq \infty$, then $M_{s^{\prime}}(\Gamma)^{\vee} \subset M(\infty, s: \infty) \subset\left(L^{s}, l^{\infty}\right)(G)$ and $\|\check{\mu}\|_{s \infty} \leq\|\| \mu\left\|\mid \leq C^{2}\right\| \mu \|_{s^{\prime}}$ where $C$ is a constant depending on $G$ and $s$. 
Proof. 1. Let $\left\{\psi_{U}\right\}$ be the approximate identity of $L^{1}(G)$ ) defined in [15, page 462]. Since $S_{0}(G)$ is a Segal algebra we have for $\mu \in M(\infty: p, q)$ and $h \in S_{0}(G)$ that

$$
\begin{aligned}
|\langle h, \mu\rangle| & =\lim \left|\left\langle h * \psi_{U}, \mu\right\rangle\right|=\lim \left|\left\langle h, \mu * \phi_{U}\right\rangle\right| \\
& \leq \lim \left|\left\|\mu \left|\| \| \hat { \psi } _ { U } \| _ { \infty } \| h \left\|_{p^{\prime} q^{\prime}} \leq\left|\|\mu \mid\|\|h\|_{p^{\prime} q^{\prime}}\right.\right.\right.\right.\right.
\end{aligned}
$$

By [14, Proposition 2.6] we conclude that $\mu$ is in $\left(L^{p}, l^{q}\right)$ and $\|\mu\|_{p q} \leq$ $\mid\|\mu\| \|$. The rest of the proof follows from (7) above. Part b) and 2 are proven in a similar manner.

3. Let $\mu \in M(r, s: \infty, q)$ and $h \in \Phi_{\infty 1}(G)$. As in the proof of part 1 using [14, Theorem 1.6] we have that

$$
\begin{aligned}
|\langle h, \mu\rangle| & \leq \lim \left|\left\langle\psi_{U}, \mu * h\right\rangle\right| \leq \lim \left\|\psi_{U}\right\|_{1 q^{\prime}}\|\mu * h\|_{\infty q} \\
& \leq\|\mu * h\|_{\infty q} \leq\|\mid \mu\|\|\| \hat{h}\left\|_{r s} \leq\right\| \mu\|\| C\|h\|_{s^{\prime} r^{\prime}} .
\end{aligned}
$$

By the density of $\Phi_{\infty 1}$ in $\left(L^{s^{\prime}}, l^{r^{\prime}}\right)(G)$ [14, Proposition 2.5] we conclude that $\|\mu\|_{r s} \leq\|\| \mu\|\|$. The rest of the proof follows from (7) above. The proofs of 4 and 5 are similar.

\section{References}

[1] L. Argabright, and J. Gil de Lamadrid, 'Fourier analysis of unbounded measures on locally compact Abelian groups', Mem. Amer. Math. Soc. 145 (1974).

[2] J. P. Bertandias and C. Dupuis, 'Transformation de Fourier sur les espaces $l^{p}\left(L^{p}\right)$ ', Ann. Inst. Fourier (Grenoble) 29 (1979), 189-206.

[3] N. Bourbaki, Elements de Mathematique. Integration, Act. Sci. et Ind. 1175 (Hermann et Cie, Paris, 1973).

[4] H. G. Feichtinger, 'Un espace de Banach de distributions tempérées sur les groupes localement compacts Abeliens', C. R. Acad. Sci. Paris Sér. I Math. 290 (1980), 791-794.

[5] H. G. Feichtinger, 'On a new Segal algebra', Mh. Math. 92 (1981), 269-289.

[6] A. Figá-Talamanca, 'On the subspace of $L^{p}$ invariant under multiplication of transform by bounded continuous functions', Rend. Sem. Mat. Univ. Padova 35 (1965), 176-189.

[7] A. Figá-Talamanca, and G. I. Gaudry, 'Multipliers and sets of uniqueness of $L^{p}$, Michigan Math. J. 17 (1970), 179-191.

[8] J. J. F. Fournier, 'On the Hausdorff-Young theorem for amalgams', Math. 95 (1983), 117-135.

[9] J. J. F. Fournier and J. Stewart, 'Amalgams of $L^{p}$ and $l^{q}$, Bull. Amer. Math. Soc. 13 (1985), 1-21.

[10] F. Holland, 'On the representations of functions as Fourier transforms of unbounded measures', Proc. London math. Soc. (3) 30 (1975), 347-365.

[11] R. Larsen, An introduction to the theory of multipliers, Grundlehren Math. Wiss. 175 (Springer, Berlin, 1971).

[12] J. Stewart, 'Fourier transforms of unbounded measures', Canad. J. Math. 31 (1979), 1281-1292. 
[13] M. L. Torres de Squire, 'Amalgams of $L^{p}$ and $l^{q}$, (Ph. D. Thesis, McMaster University, Canada, 1985).

[14] M. L. Torres de Squire, 'Multipliers for amalgams and the algebra $S_{0}(G)$ ', Canad. $J$. Math. 39 (1987), 123-148.

[15] M. L. Torres de Squire, 'Resonance classes of measures', Internat. J. Math. Math. Sci. 10 (1987), 461-472.

Department of Mathematics and Statistics

University of Regina

Regina

Saskatchewan

Canada S4S 0A2 